\title{
Avaliação da Abordagem Fisioterapêutica no Tratamento de Paciente Pediátrico Portador de Mielomeningocele
}

\author{
Fabiana Azevedo Santos \\ Centro de Tratamento e Pesquisas em Fisioterapia, Rio de Janeiro, \\ fabianafisioterapiarjeyahoo.com.br
}

Resumo: mielomeningocele é uma anomalia congênita complexa da coluna que causa vários graus de malformação da coluna espinal, ou mielodisplasia. É comumente referida como espinha bífida e é classificada como um defeito do tubo neural. Pacientes com essa desordem apresentam-se com um espectro de imperfeições, mas os déficit funcionais primários são a paralisia da coluna lombar e a perda sensorial, disfunção da bexiga e do intestino, e a disfunção cognitiva. Problemas médico-cirúrgicos e de reabilitação surgem no paciente com mielomeningocele desde o nascimento até a idade adulta. Esse trabalho apresentará a avaliação da terapia física adaptada no tratamento de pacientes pediátricos que possuam mielomelingocele.

Palavras-chave: Mielomelingocele. Mielodisplasia. Fisioterapia. Pediatria.

\section{Avaluation of The Physiotherapic Approach Inthe Treatment of Pediatric Patient That Has Myelomeningocele}

\begin{abstract}
: myelomeningocele is a complex congenital spinal anomaly that causes varying degrees of spinal cord malformation, or myelodysplasia. It is commonly referred as spina bifida and is classified as a defect of the neural tube. Patients with this disorder present with a spectrum of impairments, but the primary functional deficits are lower limp paralysis and sensory loss, bladder and bowel dysfunction, and cognitive dysfunction. Medical surgical, and rehabilitation issues arise in the patient with myelomeningocele from birth through adulthood. This work will present the evaluation of the physical therapy approach in the treatment of pediatric patients that has myelomeningocele.
\end{abstract}

Keywords: Myelomeningocele. Myelodysplasia. Physiotherapy. Pediatry 
Avaliação da Abordagem Fisioterapêutica no Tratamento de Paciente Pediátrico Portador de Mielomeningocele

Fabiana Azevedo Santos

Reabilitação e Neurologia

\section{Introdução}

As doenças que envolvem o tubo neural são responsáveis por um número considerável de pacientes a serem tratados com fisioterapia. Dentre essas doenças, encontramos a mielomeningocele, que é uma forma de disrafismo espinhal, ocasionada por falha na fusão dos arcos vertebrais posteriores e displasia (crescimento anormal) da medula espinhal e membranas que a envolvem, provocando uma deficiência neurológica (sensitiva e motora) abaixo da lesão que pode gerar paralisias e hipoestesias de membros inferiores [Vinck et al., 2009; Thompson, 2009].

Essa patologia é a segunda maior causa de deficiências crônicas do aparelho locomotor em crianças. Tem a etiologia desconhecida pela maioria dos autores, mas estudos recentes associam fatores como: genética, ambiente e nutrição [McLone, 1996; Fletcher et al., 2005; Lindquist et al., 2009].

Os sintomas dependem da localização e do grau de extensão da medula espinhal, se manifestam através de alterações motoras, sensitivas, tróficas e esfincterianas como diminuição da força muscular, paralisia flácida, hidrocefalia, incontinência dos esfíncteres do reto e bexiga [Dias, 2005; Vinck et al., 2006; Lindquist et al., 2009].

O prognóstico está ligado ao nível de lesão e se agrava com a presença de hidrocefalia, deformidades da coluna ou lesões adicionais. $O$ diagnóstico pode ser feito ainda intrauterinamente, o que aumenta as chances de tratamento. Servem de exames complementares: radiografia simples, tomografia computadorizada e ressonância magnética; que identificam malformações anatômicas [CDCP, 2004].

O tratamento engloba uma equipe multidisciplinar, pois os problemas decorrentes não podem ser tratados isoladamente. Há a necessidade de um tratamento cirúrgico para fechamento da lesão, implantação de um shunt ventrículo-peritoneal para drenar o líquido cefalorraquidiano do ventrículo lateral ao peritônio, onde será reabsorvido [Hetherington et al., 2006; Ausili et al., 2008].

\section{Tratamento}

Abordaremos o tratamento fisioterapêutico, de elevada importância na recuperação dos sintomas instalados, que tem como objetivos, promover independência funcional, prevenir deformidades secundárias, prevenir úlceras de decúbito, prevenir deficiências cognitivas secundárias, corrigir deformidades, promover aprendizado das habilidades motoras, ajustes posturais, deambulação independente ou treinamento do uso de cadeira de rodas, fortalecimento muscular, enfim, promover qualidade de vida durante as fases de desenvolvimento dessa criança [Ausili et al., 2008].

A anamnese deve ser realizada com todo o cuidado e atenção, para promover um tratamento precoce e atrativo à criança. Ao avaliar uma criança com esta patologia, deveremos abordar larga possibilidade de testes, como, teste muscular manual para determinar a extensão da paralisia motora; avaliação da amplitude de movimento para identificação de possíveis contraturas presentes; teste de reflexos em que se verifica a presença de atividade reflexa normal e a integração de reflexos primitivos e suas reações

Rev. de Saúde, Vassouras, v. 1, n. 1, p. 01-06, jan./mar., 2010 
Avaliação da Abordagem Fisioterapêutica no Tratamento de Paciente Pediátrico Portador de Mielomeningocele

Fabiana Azevedo Santos

Reabilitação e Neurologia

mais maduras; avaliação do desenvolvimento funcional para verificar se há componentes normais de postura, padrões de movimento e mobilidade; avaliações perceptivas e cognitivas que abrangem escalas próprias relacionadas à idade da criança [Greve et al, 2001; Verhoeff et al., 2006]

O tratamento deve começar rapidamente e ser dividido de acordo com as fases do indivíduo.

Período neonatal - É importante o fisioterapeuta avaliar a sensibilidade, motricidade, postura em repouso, movimentos ativos, anormalidades, deformidades e reflexos, para programar um programa de reabilitação adequado, pois o desenvolvimento do paciente tende a ficar prejudicado, devido ao fato de a criança permanecer em hospitais, para fazer as cirurgias necessárias. Logo, os estímulos são importantíssimos e devem ser feitos diariamente, até pela mãe, que ao carregar a criança de maneira correta, começa a estimular o controle cervical. Nessa fase, as deformidades mais esperadas são o pé eqüino varo e a luxação congênita de quadril. Uma das deformidades mais graves, encontrada em grande número nos lactentes, é a cifose que se manifesta desde o nascimento, na região correspondente à espinha bífida, e se agrava enquanto a criança cresce, acima da cifose se desenvolve a lordose compensatória. Qualquer movimento presente deve ser observado, estimulado, e se houver qualquer sensação nos membros inferiores, isto pode ser usado para estimular o movimento, através da palpação e toques. Os movimentos de membros superiores podem ser iniciados assim que a criança responde e exercita o estiramento da cintura escapular, que é gradativamente introduzido [Mancini et al., 2002].

Período Pré-escolar - O fisioterapeuta deve pesquisar os grupamentos musculares ativos para ter conhecimentos dos músculos que poderão utilizar para promover a independência e de quais recursos precisará. A criança deve ser estimulada diariamente e colocada em todas as posições para promover desenvolvimento das demais musculaturas. As dificuldades de percepção, função de mão e indiscriminação de lateralidade são responsáveis também por dificultar a deambulação, portanto, estratégias devem ser implementadas, como, colocar a criança em posição ortostática, ainda que a marcha não seja atingida. A abolição da sensibilidade nem sempre corresponde à deficiência da motricidade, costuma ser difícil determiná-la exatamente, e acarreta dois problemas: risco de traumatismo dos tecidos moles, devido à incapacidade de perceber dor, temperatura e pressão e perda das informações e da retroalimentação normalmente transmitidas pelos diversos órgãos dos sentidos [Lopes et al., 2009].

Período Escolar - A criança pode preferir usar a cadeira de rodas para facilitar seu deslocamento, e desse modo, cabe ao fisioterapeuta ensinar-lhe as habilidades necessárias para a sua independência. Outra questão a ser discutida é a incontinência de reto e bexiga, pelo fato de a bexiga nunca se esvaziar completamente, o que resulta em a urina começa a gotejar e a criança não perceber, se isso ocorrer, deve-se treinar a criança para a realização da drenagem vesical. O treinamento da evacuação também é bem sucedido, pois o paciente passa a ser capaz de evacuar em intervalos regulares [Long and Cintas, 2009].

Rev. de Saúde, Vassouras, v. 1, n. 1, p. 01-06, jan./mar., 2010 
Avaliação da Abordagem Fisioterapêutica no Tratamento de Paciente Pediátrico Portador de Mielomeningocele

Fabiana Azevedo Santos

Reabilitação e Neurologia

Adolescência - Nessa fase, os problemas associados à coluna vertebral voltam a preocupar e passa a ser necessário o uso de órteses [Stokes, 2000].

Fase adulta - Nessa fase, os profissionais que acompanharam o paciente até aqui, passam apenas a espectadores e realizam algumas orientações e adaptações, quando necessárias [Stokes, 2000].

\section{Conclusão}

A mielomeningocele permite às crianças atingir a fase adulta, logo, é de extrema valia uma equipe multidisciplinar, para proporcionar qualidade de vida e amenizar os danos a que estão suscetíveis. O desempenho motor da criança paraplégica deve ser considerado como sendo adaptativo e residual e não como deficiente. Muitas Pesquisas seriam favoráveis na buscar de adaptações mais eficazes para certos músculos e conse aprendizagem dos comportamentos adequados. 
Avaliação da Abordagem Fisioterapêutica no Tratamento de Paciente Pediátrico Portador de Mielomeningocele

Fabiana Azevedo Santos

Reabilitação e Neurologia

\section{Referências}

Ausili E., Focarelli B, Tabacco F, Fortunelli G, Caradonna P, Masssimi L, et al. Bonemineral density and body composition in a myelomeningocele children population: effects of walking ability and sport activity. Eur Rev Med Pharmacol Sci. 2008;12:349-354.

CDCP, Centers for Disease, Control and Prevention. Spina bifida and anencephaly before and after folic acid mandate - United States, 1995-1996 and 1999-2000. MMWR Morb Mortal Wkly Rep. 2004; 53:362-365.

Dias MS. Neurosurgical causes of scoliosis in patients with myelomeningocele: an evidence-based literature review. J Neurosurg. 2005; 103:24-25.

Fletcher JM, Copeland K, Frederick JA et al. Spinal lesion level in spina bifida: a source of neural and cognitive heterogeneity. J Neurosurg. 102:268-279, 2005.

Greve et al. Diagnóstico e Tratamento da Lesão Medula Espinhal. $1^{\mathrm{a}}$ ed. São Paulo: Roca, 2001;

Hetherington R, Dennis M, Barnes M, et al. Functional outcome in young adults with spina bifida and hydrocephalus. Childs Nerv Syst. 2006;22:117-124.

Lindquist B, Uvebrant P, Rehn E, Carlsson G. Cognitive functions in children with myelomeningocele without hydrocephalus. Childs Nerv Syst. 2009

Long TM. \& Cintas HL. Manual de Fisioterapia Pediátrica. Revinter, Rio de Janeiro, 2001.

Lopez, Fábio Ancona, Jr, Dioclécio Campos. Tratado de Pediatria. Editora Manole, 2009, São Paulo.

Mancini MC, Teixeira S, Araújo LG, Paixão ML, Magalhães LC, Coelho ZAC, et al. Estudo do desenvolvimento da função motora aos 8 e 12 meses de idade em crianças nascidas pré-termo e a termo. Arq Neuropsiquiatr. 2002; 60(4): 974-80.

McLone DG. Myelomeningocele. In: Youmans JR. Neurological surgery. Philadelphia:WB Saunders; 1996. p.843-60

Stokes M. Neurologia para Fisioterapeuta. 1a ed. São Paulo: Editorial Premier: 2000.

Thompson DN. Postnatal management and outcome for neural tube defects including spina bifida and encephalocoeles. Prenat Diagn. 2009

Verhoef M, Bart HA, Post MW, et al. Functional independence among young adults with spine bifida, in relation to hydrocephalus and level of lesion. Dev Med Child Neurol. 2006;48:114-119

Vinck A, Maassen B, Mullaart R, Rotteveel J. Arnol- Chiari malformation and cognitive functioning in spina bifida. J Neurol Neurosurg Psychiatry. 2006;77(9):1083-6.

Vinck A, Niijhuis-van der Sanden MW, Roeleveld NJ, Mullaart RA, Rotteveel JJ, Maassen BA. Motor profile and cognitive functioning in children with spina bifida. Eur J Paediatr Neurol. 2009

Rev. de Saúde, Vassouras, v. 1, n. 1, p. 01-06, jan./mar., 2010 